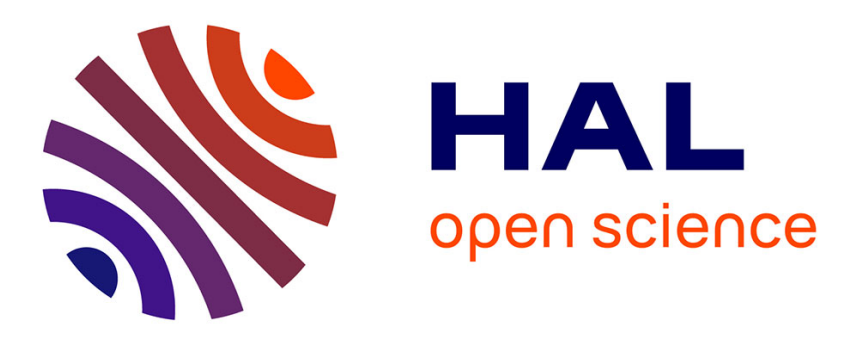

\title{
Alteration of Asian lacquer: in-depth insight using a physico-chemical multiscale approach
}

Anne-Solenn Le Hô, Chloé Duhamel, Céline Daher, Ludovic Bellot-Gurlet, Céline Paris, Martine Regert, Michel Sablier, Guilhem André, Paul Desroches, Paul Dumas

\section{To cite this version:}

Anne-Solenn Le Hô, Chloé Duhamel, Céline Daher, Ludovic Bellot-Gurlet, Céline Paris, et al.. Alteration of Asian lacquer: in-depth insight using a physico-chemical multiscale approach. Analyst, 2013, 138, pp.5685 - 5685. 10.1039/c3an00608e . hal-01481496

\section{HAL Id: hal-01481496 https://hal.science/hal-01481496}

Submitted on 2 Mar 2017

HAL is a multi-disciplinary open access archive for the deposit and dissemination of scientific research documents, whether they are published or not. The documents may come from teaching and research institutions in France or abroad, or from public or private research centers.
L'archive ouverte pluridisciplinaire HAL, est destinée au dépôt et à la diffusion de documents scientifiques de niveau recherche, publiés ou non, émanant des établissements d'enseignement et de recherche français ou étrangers, des laboratoires publics ou privés. 


\title{
PAPER
}

Cite this: Analyst, 2013, 138, 5685

\section{Alteration of Asian lacquer: in-depth insight using a physico-chemical multiscale approach $\uparrow$}

\author{
Anne-Solenn Le Hô, ${ }^{* a}$ Chloé Duhamel, ${ }^{a}$ Céline Daher, ${ }^{a}$ Ludovic Bellot-Gurlet, ${ }^{b}$ \\ Céline Paris, ${ }^{\mathrm{b}}$ Martine Regert, ${ }^{\mathrm{C}}$ Michel Sablier, ${ }^{\mathrm{d}}$ Guilhem André, ${ }^{\mathrm{e}}$ Jean-Paul Desroches ${ }^{\mathrm{e}}$ \\ and Paul Dumas ${ }^{\dagger}$
}

Oriental lacquer has been used in Asian countries for thousands of years as a durable and aesthetic coating material for its adhesive, consolidating, protective and decorative properties. Although these objects are made from an unusual material in Occident, Western museum collections host many lacquerwares. Curators, restorers and scientists are daily confronted with questions of their conservation and their alteration. The characterization of their conservation state is usually assessed through visual observations. However deterioration often starts at the microscopic level and cannot be detected by a simple visual inspection. Often, ageing and deterioration of artworks are connected to physical, mechanical and chemical transformations. Thus new insight into alteration of lacquer involves the monitoring of macro-, microscopic and molecular modifications, and this can be assessed from physicochemical measurements. Non-invasive (microtopography and Scanning Electron Microscopy - SEM) and micro-invasive (infrared micro-spectroscopy using a synchrotron source - SR- $\mu \mathrm{FTIR}$ ) investigations were performed to study the degradation processes of lacquers and evaluate their level of alteration. In particular, spectral decomposition and fitting procedure were performed in the $1820-1520 \mathrm{~cm}^{-1}$ region to follow the shift of the $\mathrm{C}=\mathrm{O}$ and $\mathrm{C}=\mathrm{C}$ band positions during lacquer ageing. The present work proves the potential of this physico-chemical approach in conservation studies of lacquers and in the quantification of the state of alteration. It evidences chemical phenomena of alteration such as oxidation and decomposition of a lacquer polymeric network. It also demonstrates for the first time the degradation front of artificially aged lacquer and the chemical imaging of a more than 2000 years old archaeological lacquer by using SR- $\mu$ FTIR.

Received 28th March 2013

Accepted 10th July 2013

DOI: 10.1039/c3an00608e

www.rsc.org/analyst optical attributes and also the diversity of its functions (coating, decoration, adhesiveness) have evolved incessantly through time and geographical areas in Asia. ${ }^{1}$

Asian lacquer is a vegetal emulsion extracted from trees of the Anacardiaceae family, which grow in Asia. When the sap is applied in several micrometric layers, up to several tenths, on a substrate, it polymerizes and forms a durable gloss coating which is resistant to water, most solvents, bacteria, etc. and provides a nice decorative effect. The foundation layers usually consist of lacquer mixed with many coarse substances as clay, flour, rice starch paste, sawdust, linen, etc., while the intermediate and finishing layers exhibit gradually finer granulometry. The composition of the latter is also variable, as they can consist of a combination of lacquer with pigments and metallic particles or foils. ${ }^{1}$

This coating produces a background, which is often highlighted by painting with lacquer, surface relief works, inlaid elements (metal, pearl, eggshells, ivory, etc.) and metallic leaf or powders. The viscoelastic property of lacquer also enables it to be carved, engraved or incised. ${ }^{2}$ At the end of the process, a lacquer ware is obtained which is a heterogeneous and 
multi-layered system due to the successive application of thin layers. Cross-sections are very useful to investigate the different layers, their spatial organization, composition, thickness and particle size distribution. ${ }^{3}$

Asian lacquer is a complex material with a high structural hierarchy from the macro- to the nanoscale. From a chemical point of view, freshly collected lacquer sap is a water in oil (W/O) emulsion. The water phase consists of a mixture of hydrophilic mono-, oligo- and polysaccharides (5-6\%), with branched structures which increase the durability of the lacquer film once cured, and enzymes (1\%) responsible for the polymerization. The oily fraction is made of substituted catechol (60-70\%), i.e. dihydroxybenzene derivatives substituted in the 3 or 4-position with saturated and unsaturated linear aliphatic side chains and a small proportion of phenol (3\%). The sap also contains waterinsoluble glycoproteins $(2-3 \%)$. Both polysaccharide and glycoprotein constituents of the sap are thought to play an important role in the structure of the lacquer matrix. ${ }^{2}$

The polymerization by enzyme-catalyzed and oxidative reactions of the sap leads to the formation of a lacquer film (Fig. 1). Catechols couple in the presence of oxygen to produce dimerized compounds such as biphenyls and dibenzofurans that indicate the onset of polymerization. The dried lacquer film presents a densely packed grain structure, called the core-shell structure (Fig. 2). Each spherical grain (1 to $10 \mu \mathrm{m}$ in diameter) is composed of a thin shell of polysaccharides (3 to $4 \mathrm{~nm}$ of thickness) and glycoproteins as adhesive surrounding the polymerized catechol. ${ }^{2}$ Such a core-shell structure is believed to be responsible for the durability of the lacquer film as long as the polysaccharide shell acts as a high barrier against oxygen.

Western museums host a large number of lacquered objects (utilitarian items, paintings, sculptures, or architectural elements) originating from numerous importations due to the opening of maritime roads between Europe and Asia, since the end of the fifteenth century. Despite their occurrence in museum collections, their preservation is still suffering from lack of knowledge and the insufficient amount of studies on alteration carried out until now. ${ }^{4-6}$ Moreover the few studies on the subject mainly dealt with the degradation of samples of model lacquer, which have no correlation with museum or archeological pieces. ${ }^{7-13}$ Yet, even if the resistance and durability of lacquer has been demonstrated in Asia with the excellent condition of some archaeological remains, lacquer objects suffer from alteration under the effect of inappropriate relative humidity or light exposure. So Asian lacquer wares can be subject to damages due to poor environmental conditions, leading to discoloration, fading, cracking, lifting lacquer, etc. Two identified major problems with lacquer wares are their<smiles>[R]c1cccc(O/C(C=CCCCCCCCCc2cccc(O)c2O)=C/C=C/C)c1O</smiles><smiles>[R]c1c(/C(C=CCCCCCCCCc2cccc(O)c2O)=C/C=C/C)ccc(O)c1O</smiles>

$$
\begin{aligned}
& \mathrm{R}: \mathrm{C}_{15} \mathrm{H}_{25} \\
& \mathrm{R}: \mathrm{C}_{17} \mathrm{H}_{29}
\end{aligned}
$$

Fig. 1 Polymerization of catechol leading to the formation of a catechol dimer from Kumanotani.2 
poor UV stability and their brittleness under dry conditions. Poor UV resistance causes permanent surface deterioration. The surface of lacquer becomes dull and faded with the formation of a network of cracks. ${ }^{7,10}$ The lacquer coating network degrades in the unsaturated side chain and oxidizes from the surface. ${ }^{8}$ Inappropriate conditions of relative humidity cause chemical and structural deterioration. Under dry conditions, the lacquer becomes brittle as water is released, decreasing the toughness and flexibility of the film. Moreover when the substrate shrinks or swells due to changes in relative humidity, the lacquer cannot accommodate itself to these dimensional changes. This results in de-cohesion of the coating from the substrate, separation of the lacquer layers, material losses and surface cracks. ${ }^{9,12-14}$

These problems of lacquer preservation are the most frequent ones found in the West, since climatic conditions are different - generally dryer - compared to the ones in Asia. The preservation of lacquered objects in the West therefore requires an accurate knowledge of their composition, ${ }^{\mathbf{1 5}}$ and also an understanding of their phenomenon of degradation in order to determine their operating mechanisms as well as the factors from which they must be protected.

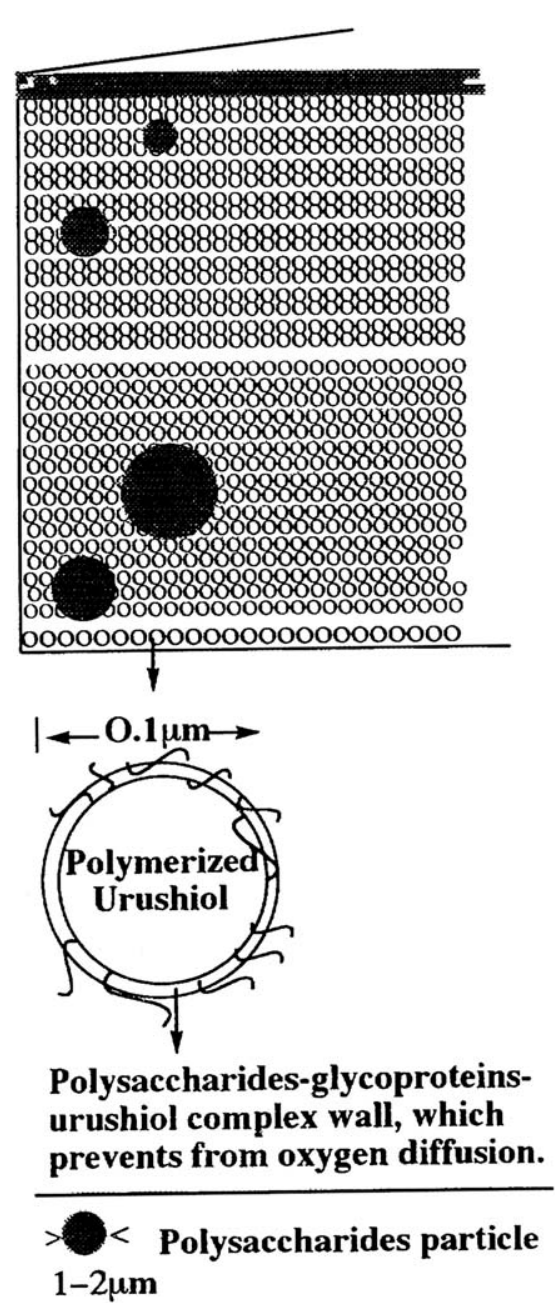

Fig. 2 The core-shell structure of a polymerized lacquer film from Kumanotani. ${ }^{23}$
In many cases, the alteration of lacquerwork is identified by simple visual observation. There is a strong need to combine visual observations of degradation to chemical or physical modifications in order to understand the deterioration mechanisms and register the first levels of damage. The latter point is of prime importance because the first levels do not necessarily affect the visual appearance of lacquers, while the first chemical and physical degradation reactions already occur. The early detection of alteration is essential in the preservation process.

This paper is focused on the assessment of the degradation of Asian lacquer based on a multi-analytical methodology. It investigates optical, chemical and structural modifications of the lacquer polymer for a better understanding of the mechanisms that occur, and to evaluate the degree of alteration in relation with the preservation status. The originality of this work is the analytical procedure, which allows multi-scale and stratigraphic investigations.

We used complementary non- and micro-destructive techniques, required for the study of precious and irreplaceable artworks. Reference multi-layers of lacquer were prepared on a wooden substrate and subjected to accelerated ageing under light exposure. The degradation in terms of surface changes, morphological characteristics, roughness, oxidation or decomposition of the polymerized network was investigated at macro-, microscopic and molecular levels by means of microtopography, SEM and SR- $\mu$ FTIR. As the IR micro-spectroscopy provides spatially resolved identification and chemical imaging in the micrometer range on the modification of the molecular information and of the structure within the build-up of the lacquer layers, cross-sections were prepared using a protocol optimized to allow the preservation of the spatial arrangement of the wood and the lacquer layers. A part of the SR- $\mu$ FTIR results here are based on the decomposition of $\mathrm{C}=\mathrm{O}$ and $\mathrm{C}=\mathrm{C}$, and $\mathrm{CH}$ vibration regions and on the study of the position of their constitutive bands. Besides it was possible to image the structure of a more than 2000 years old lacquer at the microscale. Until now, the few studies performed on the degradation of Asian lacquer by IR were carried out on modern lacquer films artificially aged without investigations correlating the spatial distribution of different phases., ${ }^{\mathbf{4 , 8 , 1 0 , 1 1}}$ Besides, analyses consisted only in global approaches conducted at the surface of the degraded film with no information on the chemical composition or the stratigraphy of a multi-layered lacquer on the microscale.

\section{Experimental set-up}

\section{Sample boards}

As wood is by far the most common material used in Asia as a substrate for lacquer, twelve sample boards made of Japanese coniferous wood and lacquer layers were prepared. These sample boards were considered as references simulating lacquer ware. A lacquer restorer and a lacquer artist, using traditional methods and similar curing layers, conjointly produced them in 2008.

Four types of lacquers - ki-, nuro-, kuro- and roiro urushiwere used in the preparation of sample boards. Ki-, kuro- and 
roiro urushi were purchased from Watanabe-Shoten (Tokyo, Japan) and nuro urushi was prepared. These different lacquers were used for specific purposes. Ki urushi is a basic raw lacquer product. It is harvested from Chinese lacquer trees and do not require any refinement. This raw lacquer dries fast and is quite hard, making it very useful for foundation layers. Nuro urushi was prepared by mixing $k i$ urushi with a rice starch paste (from Watanabe-Shoten, Tokyo, Japan). This mixture provides a strong adhesive agent used for ground layers. From the Chinese ki-urushi, moisture is evaporated by stirring and cooking it in an open vessel to produce the following processed lacquers: mixing with black pigments (iron oxides and charcoal) and drying oil makes kuro urushi and mixing with iron hydroxide makes roiro urushi. The former gives a black layer and the latter gives a brilliant and high quality lacquer which is used for top layers.

Moreover fillers - jinoko, a coarse grind of earth and tonoko, a very fine clay - combined with lacquer were used in the foundation layers to smooth imperfections and ridges of the wooden substrate. Once mixed with ki urushi, it is known as sabi. ${ }^{1}$ Jinoko and tonoko were purchased from Watanabe-Shoten (Tokyo, Japan).

Wooden Japanese coniferous plaques measuring $12 \times 12 \times$ $1 \mathrm{~cm}$ were coated of four foundation layers (ki urushi, jinoko and nori urushi, jinoko and sabi, and tonoko and ki urushi), three intermediate layers of kuro urushi and four finishing layers of roiro urushi. A spatula made of Japanese cypress was used for the application of foundations layers, while the intermediate and finishing layers were spread with a brush made from human hair (Watanabe-Shoten, Tokyo, Japan). After the application of each layer, the sample boards were put into a drying cabinet under a relative humidity of $70-80 \%$ at $25^{\circ} \mathrm{C}$ for $24 \mathrm{~h}$ to promote the hardening of the lacquer. Once dry, the lacquer film was polished with Magnolia or Camellia charcoal before the next layer was applied. The complete preparation process lasted three months.

\section{Archaeological samples}

A fragment of an archaeological lacquer was analyzed (inv. GM.05.20.74.79). It originates from a Mongolian lacquered wooden chariot. It was collected by the Mission Archéologique Française en Mongolie (MAFM) during the excavation of the tomb T20 of the Xiongnu necropolis at Gol Mod (Central Mongolia). It dated between the $1^{\text {st }}$ century B.C. and the $1^{\text {st }}$ century A.D. It presents a very altered state (de-cohesion of lacquer layers from the substrate, brittleness, etc.).

\section{Accelerated ageing}

The goal of the ageing tests was to create degraded conditions inducing an alteration of modern multi-layered sample boards under light exposure. In each case, we tried to approach the conditions of ageing of the archeological lacquer chariot using a "simplified" environment. It should be a too complex and illusive undertaking to reproduce the degradation of the chariot resulting from a long period of undefined burial (in terms of temperature, relative humidity, and interactions with its environment).

The sample boards were exposed for 4 weeks to an Osram metal halide lamp (similar to the sunlight spectrum) in a
Servathin climatic chamber equipped with an Atlas Solar source. The lamp power was $1500 \mathrm{~W}$. These conditions reproduce an exposure for about 320 years at 50 lux. The chamber was fitted with fans to dissipate the heat generated by the lamp.

Every 6 days of exposure, the boards were photographed and sampled. Temperature and $\mathrm{RH}$ were controlled $\left(22{ }^{\circ} \mathrm{C}, 50 \%\right.$ $\mathrm{RH})$.

The surface of the boards was exposed to light under three different conditions: $1 / 3$ of the surface was protected by an aluminum foil (to serve as a reference), 1/3 was protected by an UV filter (with a cut-off filter at $400 \mathrm{~nm}$ to isolate the boards from wavelengths below $400 \mathrm{~nm}$ ) and the last 1/3 was uncovered.

\section{Preparation of thin sections}

Fragments of the modern replicas and of the archaeological lacquer were prepared for the SR- $\mu$ FTIR analyses. First, samples were collected from the sample boards and archeological lacquer perpendicularly to the surface, with a scalpel. Collected samples contained both wood and multi-layered lacquer. Then, transverse thin sections with a thickness of $2 \mu \mathrm{m}$ were realized directly on the sample with a diamond knife set in a microtome without embedding in a resin. The use of a coating resin was avoided during the sample preparation to minimize the introduction of additional chemical compounds that could interfere with the infrared signatures of the lacquer. Each thin section was thoroughly observed by optical microscopy before their SR- $\mu$ FTIR analyses. We checked that the stratigraphy was conserved without any deformation of the structure and that the section thickness was constant and reproducible between samples.

The thin sections of modern replicas presented the intact build up of the layers from the wood substrate to the top lacquer layers. Fig. 3 shows the analyzed artificially aged sample board area of a transverse thin section. No distortion was induced by the preparation and all the lacquer layers and wooden substrate were still visible. However, as archeological lacquer was very altered and brittle, it was not possible to preserve the complete stratigraphy during the preparation of thin sections. There was de-cohesion of the lacquer layers from the wooden substrate.

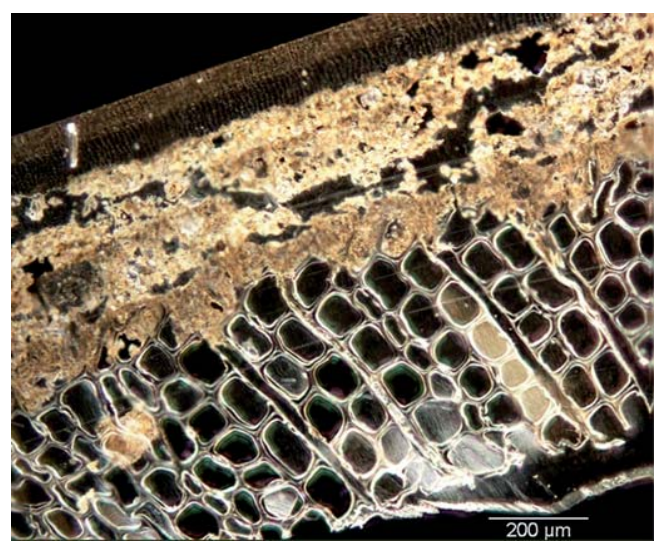

Fig. 3 Optical image of a thin section prepared from the uncovered part of a sample board after an artificial ageing for 4 weeks. The image was obtained before the SR micro-FTIR analysis at the SMIS beamline (synchrotron Soleil). 
Indeed the only lacquer layers of the archaeological remains were chemically imaged by SR- $\mu$ FTIR.

\section{Analytic}

Microtopography. This non-destructive technique allows, starting from measurements of altitude variations, the calculation of the roughness of a surface at the microscale. This technology presents an excellent vertical or lateral resolution. The microtopography is acknowledged as an accurate and reliable technique for non-contact distance and thickness measurement. It is one of the very few non-contact techniques for 3D metrology recommended by the ISO 25178 international standard.

When the lacquer degrades, macro- and micro-fissures develop. This results in a more permeable surface of the lacquer, which allows adsorption and penetration of water into it. Thus the lacquer flakes off and cracks. ${ }^{\mathbf{1 0 , 1 1 , 1 4}}$

Microtopography has been initially used to measure the roughness of lacquer and follow the propagation of cracks during ageing. Indeed, some cracks are easily visible to the naked eye; others are of microscopic scale and require the use of specific analytical techniques in order to be quantitatively characterized.

Microtopography consists of confocal measurement with an extended field of chromatic coding. The used source of white light (polychromatic) does not require preparation of the sample. Moreover, the confocal geometry allows deep optical cuts of any object. No contact with the surface takes place during the measurement therefore it is non-invasive. This is also the reason why this approach was chosen and it has enabled us to work directly on the original studies without risk of their deterioration. Furthermore, contrary to SEM, microtopography allows us to study a wider size range of the treated samples (0-20 cm of diameter).

During the measurement, the monochromatic components of the polychromatic light are separated thanks to the axial chromatism lens. When the light sweeps the surface of the sample, only the monochromatic component, which goes in contact with it, is reflected. It is therefore possible to know the altitude measurements, depending on the length of the reflected wave (retro diffusion) (Fig. 4). Thus, a rough cross-section of the surface is determined. A Gaussian filter with a $0.25 \mathrm{~mm}$ cutoff (which means that all bumps on the surface of a smaller diameter are not taken into account) allows suppression of all imperfections linked to the environment of measurement. From the obtained profile, the parameters of roughness have been calculated. For that reason, a line of reference of least squares, in other words a line to which the statistical parameters (here it is roughness) characterizing the profile of a surface are determined, is drawn on this profile. This line divides the profile in a way that inside the base length, the sum of square deviation between the peaks and troughs measured from this line is minimal.

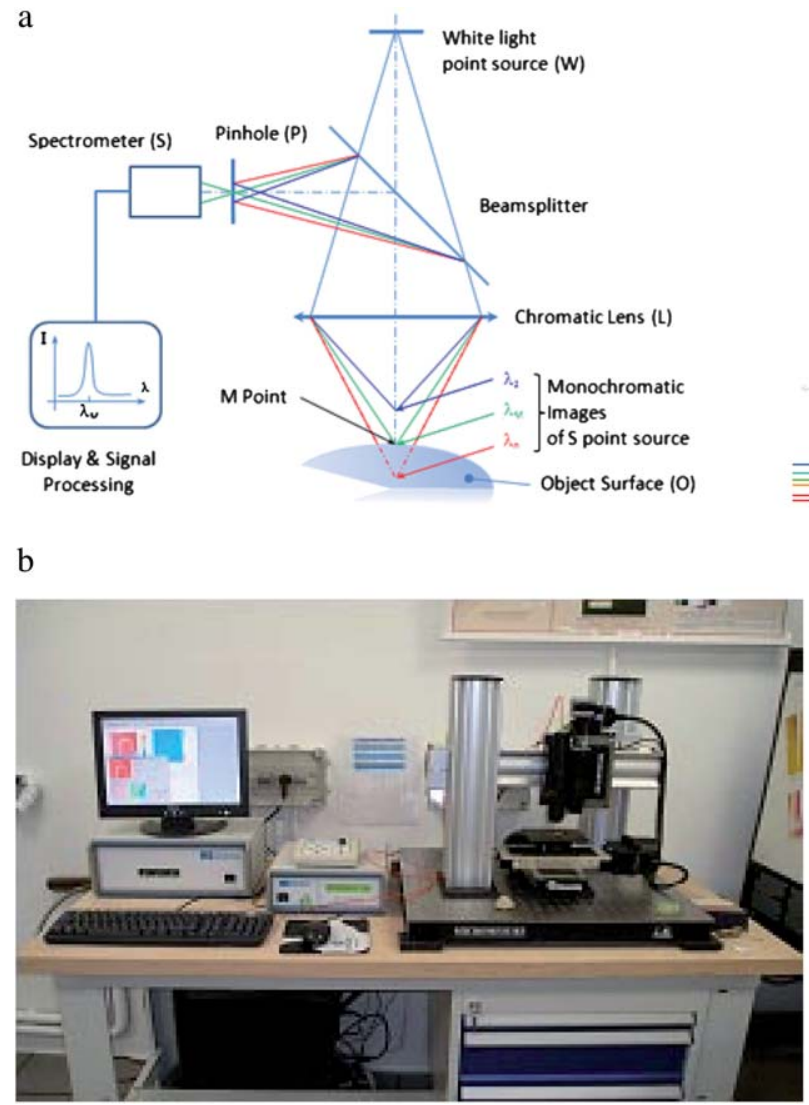

Fig. 4 (a) Principle of microtopography from STIL website (http://www.stilsa.com/index.htmv) and (b) photography of the STIL microtopography station. 
The two-dimensional root-mean-square deviation of the profile, $R_{\mathrm{q}}$, which demonstrates the root-mean-square value of the profile deviation compared to the line of reference of least squares, is expressed as

$$
R_{\mathrm{q}}=\mathrm{RMS}=\sqrt{\frac{1}{N} \sum_{i=1}^{N} y_{i}^{2}}
$$

$N$ represents the number of deviations taken into account.

Finally, the $S_{\mathrm{q}}$ parameter was also calculated. It consists of a 3D extrapolation of $R_{\mathrm{q}}$ defined according to a reference medium surface. $S_{\mathrm{q}}$ designates the three-dimensional root-mean square deviation of the surface. $S_{\mathrm{q}}$ is obtained with the rectification by the least squares plane of the measured surface, then with the alignment of altitudes around the mean, thanks to the MoutainsMap@ software (developed by STIL, Sciences et Techniques Industrielles de la Lumière). $S_{\mathrm{q}}$ is calculated using the formula

$$
S_{\mathrm{q}}=\sqrt{\frac{1}{N M} \sum_{x=0}^{N-1} \sum_{y=0}^{M-1} Z_{x, y}{ }^{2}}
$$

A station of STIL micro-measurements was used. Its measurement principle is based on chromatic confocal imaging. The system consists of a motorized $X Y Z$ stage, an optical pen sensor, an optoelectronic controller comprising the source and the spectrometer which is linked by a fiber optic cable to the optical pen and a PC computer for the acquisition and treatment of data. A data acquisition software SurfaceMap@ controls the system and MoutainsMap@ software (developed by STIL) enables post-processing.

Two artificially aged sample boards consisting of three different parts were measured with microtopography. In order to calculate the average roughness parameter, twelve zones were scanned on each part of these test sample boards.

The selected parameters were as follows: optical pen of $300 \mu \mathrm{m}$, frequency of $300 \mathrm{~Hz}$, scanned surface over a distance of $1.5 \mathrm{~mm}$ ( $X$-axis) $\times 1.5 \mathrm{~mm}$ ( $Y$-axis), constant speed of $1000 \mu \mathrm{m} \mathrm{s}^{-1}$ and step size of $20 \mu \mathrm{m}$ on the $X$ and $Y$-axis.

Scanning electron microscopy (SEM). Scanning electron microscopy (SEM) was performed on a modern artificially aged sample.

A PHILIPS XL30 ESEM microscope combined with an energy dispersive X-ray spectrometer (EDS) Inca (Oxford-Cambridge) was used for investigations. The surface of the sample boards was directly imaged under partial pressure without metallization to evaluate changes in surface morphology. Microsamples collected from the sample boards were also examined to measure the depth of cracks. These microsamples were taken from the part protected by aluminum, the part covered by a UV filter and the uncovered part. They were embedded in a polyester resin (Sody 33, Escil@) and then cut and polished to prepare cross-sections. They presented the complete stratigraphy (from the upper lacquer layer to the deeper wood substrate).
Synchrotron radiation micro-FTIR microspectroscopy (SR- $\mu$ FTIR). Synchrotron powered infrared micro-spectroscopy was performed at the SMIS beamline of the SOLEIL synchrotron (Saint-Aubin, France) on a Thermo Nicolet Continuum XL and NicPlan microscopes equipped with a liquid nitrogen cooled mercury cadmium telluride detector (MCT-A, $50 \times 50 \mu \mathrm{m}^{2}$ ).

The IR spectra and images were collected on thin sections deposited on MirrIR low-e silver-coated microscope slides (Kevley Technologies, USA) and measured in transflection mode with a $\times 32$, N.A. 0.68 Schwarzschild objective in confocal geometry (spectral range $4000-650 \mathrm{~cm}^{-1}$, aperture $10 \times 10$ or $8 \times 8 \mu \mathrm{m}^{2}$, step size 10 to $6 \mu \mathrm{m}, 64$ to 256 scans, spectral resolution of $4 \mathrm{~cm}^{-1}$ ).

Previous studies described and evaluated interferences in transflection mode. ${ }^{\mathbf{1 6 , 1 7}}$ This mode is more sensitive to optical effects than the transmission mode and thus can induce some spectral distortions. These previous studies were mainly conducted in the biomedical and biological fields. In this work, the cross-sections for the SR- $\mu$ FTIR analyses by transflection were prepared without evaporation of samples, as it is usually the case for biological samples. The obtained sections are flat and more homogeneous in thickness than the ones prepared from biological and histological samples. This spectral distortion effect does not contribute markedly to our study, since the refractive index of the lacquer is different from that of biological tissue. Finally a second derivative was performed to the SR- $\mu$ FTIR spectra to give more accurate results and detect changes due to ageing. This procedure enables spectral differences during ageing to be exhibited.

IR data processing. Each map was treated after a linear baseline correction using Opus software.

In some chemical images, the area ratios of bands have been generated to better study and quantify the evolution of IR bands during the alteration. The following ratios were selected according to maps: $1270 / 2855\left(\nu \mathrm{C}-\mathrm{OH} / \nu \mathrm{CH}_{2}\right), 1210 / 2855(\nu \mathrm{COC} /$ $\left.\nu \mathrm{CH}_{2}\right)$, and $990 / 2855\left(\nu \mathrm{CH}\right.$ of trienic side chain $\left./ \nu \mathrm{CH}_{2}\right)$. The band at $2855 \mathrm{~cm}^{-1}$, attributed to $\nu \mathrm{CH}_{2}$ in the catechol side chain, was selected because no change during ageing was noted. Five analyzed points were made for the part of the sample boards protected by aluminum and the uncovered one. A simple linear baseline correction was performed before the ratio calculation.

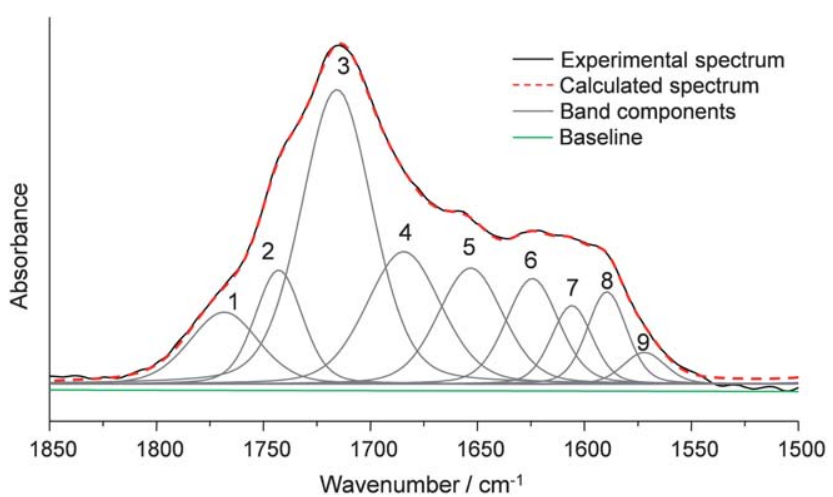

Fig. 5 Decomposition and curve fitting analysis of the SR micro-FTIR spectrum of the uncovered part of a sample board after an artificial ageing for 4 weeks. 
Moreover, some studies conducted on cultural heritage materials, such as tortoiseshell, horn, galalith, bone, resin, gum and oil, showed additional relevant parameters related to discriminating criteria, secondary structures and conservation state could be extracted from a spectral decomposition. ${ }^{18-22}$ Indeed, to go further in the interpretation of IR data and to be sufficiently sensitive to detect the first levels of alterations of lacquer, a deconvolution into 9 components was obtained for the $1820-1520 \mathrm{~cm}^{-1}$ region. We investigated this large region of interest to extract more information on the oxidation products, the reactivity of the side chain of catechols and to follow the evolution of the polymeric network.

Second-derivative functions were performed to the SR microFTIR spectra collected from the artificially aged sample boards in order to determine the number and the frequencies of the individual band components in the spectral range 1820-1520 $\mathrm{cm}^{-1}$. First, the spectra were truncated between 1820 and $1520 \mathrm{~cm}^{-1}$, a linear baseline was subtracted and normalization was performed using Opus software. Then a decomposition and curve fitting procedure permitted to fit the spectra profiles with these components. The fits were made with home-made software (named ASREL-Ajustement de spectres en raies élémentaires, and developed by the LADIR). All spectra could be fitted by nine band components (Fig. 5). The relative contributions of these components were obtained using a band-fitting procedure keeping the wave numbers, half widths and area free and band profiles constant (75\% Gaussian and 25\% Lorentzian). Four or five measurements for the different parts of the sample boards were made to check the reproducibility of the decomposition and curve fitting procedure.

\section{Results and discussion}

\section{Visual examination and SEM}

Fig. 6 shows a sample board after the artificial ageing. The part of the board covered by aluminum has an aspect similar to the one before the test. In contrast, the uncovered part presents a high decrease of brightness with an increase in the transparency. Additionally, the brightness is slightly lowered in the part protected by an UV filter, indicating an intermediate state. These visual examinations were also confirmed by gloss meter measurements (ESI Fig. s1†).

The SEM images of the different parts of the board are reported in Fig. 7. They allow us to correlate the decrease in

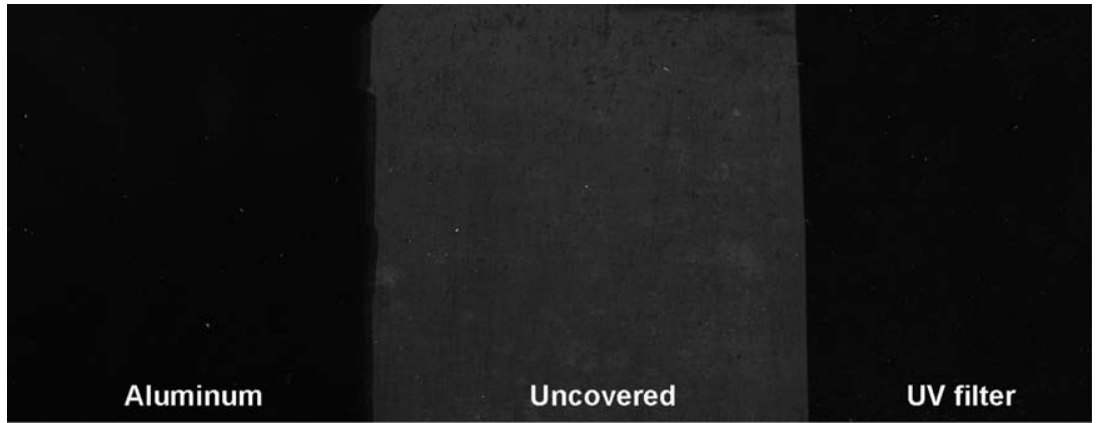

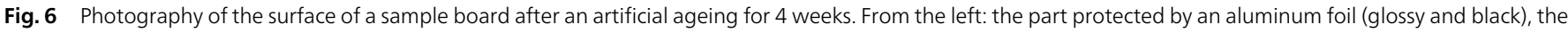
uncovered part (matt and grey), and the part protected by a UV filter (slight decrease of brightness).
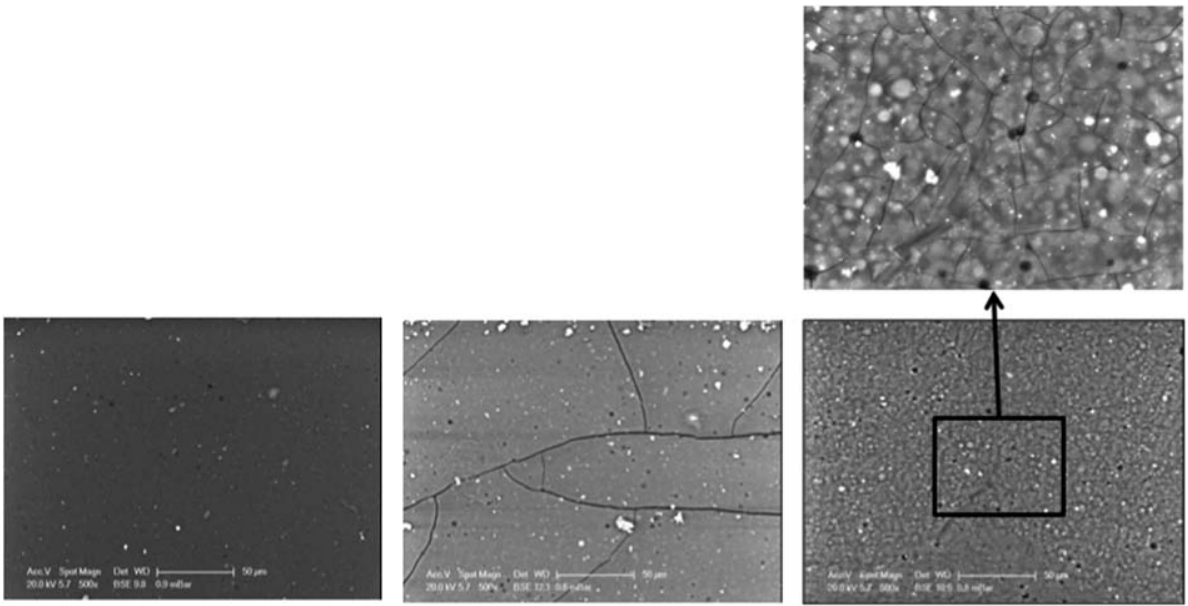

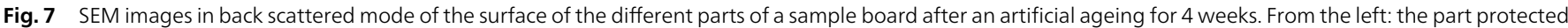

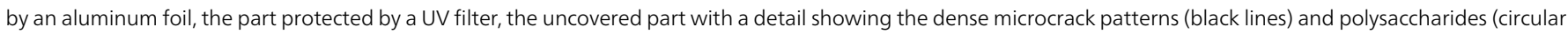
particles appearing in white contrast). 
gloss observed by a visual examination to a densification of the crack pattern. In fact, the part of the board covered by aluminum shows a smoother surface. A large crack pattern appears in the part of the board protected by a UV filter, whereas the uncovered part presents dense microcrack patterns and holes under exposure.

According to Kumanotani, the UV exposure of a lacquer film leads to deterioration of the surface and the subsequent appearance of the dispersed polysaccharide particles in the surface layer which causes a decrease in the surface gloss. ${ }^{2,23} \mathrm{We}$ suggest that the clearly observed circular particles $(1-2 \mu \mathrm{m}$ in diameter, Fig. 7) are due to dispersed polysaccharides. This observation has already been noticed in the case of deterioration of the lacquer film due to UV. ${ }^{10}$ Under light exposure, the lacquer deteriorates; subsequently the core-shell structure of the lacquer with its densely packed architecture of grains is damaged. The shell polysaccharide can be broken making the core composed of polymerized catechol more prominent ${ }^{11}$ as illustrated in the right image of Fig. 7.

To quantify these observations and the roughness relative to the decrease in the brightness, the appearance and the densification of crack patterns under the light irradiation, measurements by microtopography were performed.

\section{Measurement of roughness with the microtopographic station}

Table 1 presents the value of root-mean square deviation, $S_{\mathrm{q}}$, for the different parts of the sample boards.

The root-mean square deviation $S_{\mathrm{q}}$ is smaller when the lacquer is protected by aluminum than in the two other cases. An increase of $30 \%$ for the part of the board covered by a UV filter and an increase of more than $160 \%$ for the uncovered part were observed.

Table 1 Root-mean square deviation, $S_{\mathrm{q}}$, measured by microtopography for the different parts of the sample boards after an artificial ageing for 4 weeks

\begin{tabular}{lllll}
\hline & $\begin{array}{l}\text { Number of } \\
\text { scanned } \\
\text { surfaces }\end{array}$ & $\begin{array}{l}\text { Mean } \\
(\mu \mathrm{m})\end{array}$ & $\begin{array}{l}\text { Range } \\
(\mu \mathrm{m})\end{array}$ & $\begin{array}{l}\text { Standard } \\
\text { deviation }\end{array}$ \\
\hline $\begin{array}{l}\text { Samples } \\
\begin{array}{l}\text { Part protected } \\
\text { by an aluminum foil }\end{array}\end{array}$ & 12 & 0.099 & $0.058-0.141$ & 0.054 \\
$\begin{array}{l}\text { Part protected } \\
\text { by an UV filter }\end{array}$ & 12 & 0.138 & $0.105-0.219$ & 0.031 \\
$\begin{array}{l}\text { Uncovered part } \\
\text { Uncen }\end{array}$ & 12 & 0.28 & $0.212-0.352$ & 0.046
\end{tabular}

The higher the roughness, the less the brightness. These results therefore confirm the visual observations (Fig. 6), considering that lacquer protected by an aluminum foil is the shiniest, followed by the one protected by an UV filter and finally the lacquer that was not protected by light is matt. We have also extracted roughness profiles. The uncovered part of the sample board presents the highest deviation $(2.5 \mu \mathrm{m}$ peakto-valley). The maximum difference in height is $0.8 \mu \mathrm{m}$ when the lacquer is covered by an UV filter and $0.6 \mu \mathrm{m}$ when the lacquer is protected from light exposure by an aluminum foil.

\section{Validation}

In order to confirm the relevance of the microtopographic measurements, a SEM examination was performed on crosssections. We collected samples of each part of an artificially aged test sample board. The SEM examination allowed us to study the lateral distribution of the cracks and to measure their depth (Fig. 8). The cracks are deeper and deeper as the protection decreases. When a lacquer is not protected during ageing, the depths of the cracks are in the order of $2.5 \mu \mathrm{m}$, in agreement with the microtopographic measurements. Microtopography is, then, very useful in the frame of detection and quantification of

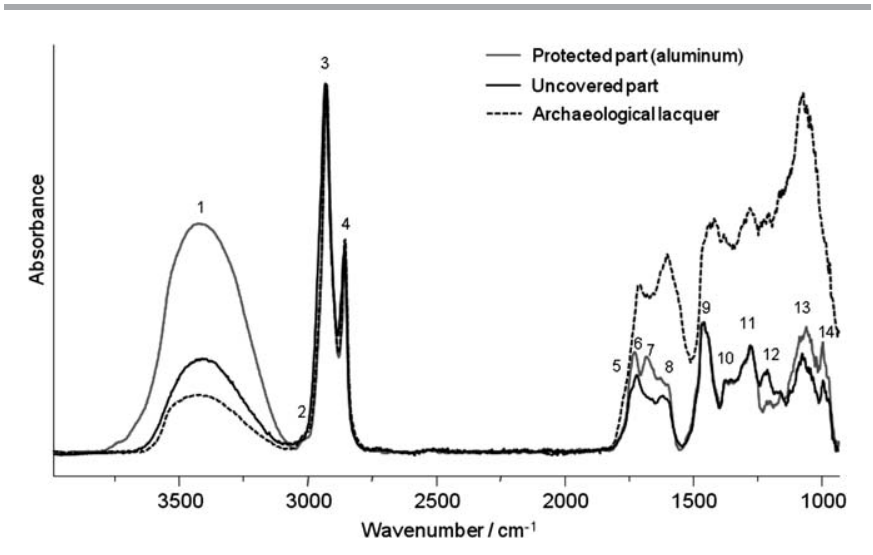

Fig. 9 SR micro-FTIR spectra of parts of a sample board protected by an aluminum foil, uncovered part after an artificial ageing for 4 weeks and an SR micro-FTIR spectrum collected from the Mongolian archaeological lacquer chariot from the Gol Mod site ( $1^{\text {st }}$ century $B C-1^{\text {st }}$ century AD). Spectra performed in transflection mode on thin sections at SMIS beamline, synchrotron Soleil (128 scans, spectral resolution of $4 \mathrm{~cm}^{-1}$, aperture $10 \times 10 \mu \mathrm{m}^{2}$, step size $10 \mu \mathrm{m}$ ). Bands labeled as follows: (1): $3400 \mathrm{~cm}^{-1},(2): 3005 \mathrm{~cm}^{-1},(3): 2930 \mathrm{~cm}^{-1}$, (4): $2855 \mathrm{~cm}^{-1}$, (5): $1725 \mathrm{~cm}^{-1},(6): 1680 \mathrm{~cm}^{-1},(7): 1625 \mathrm{~cm}^{-1},(8): 1591 \mathrm{~cm}^{-1},(9)$ : $1465 \mathrm{~cm}^{-1},(10): 1370 \mathrm{~cm}^{-1},(11): 1270 \mathrm{~cm}^{-1},(12): 1210 \mathrm{~cm}^{-1},(13): 1045 \mathrm{~cm}^{-1}$, and (14): $990 \mathrm{~cm}^{-1}$.
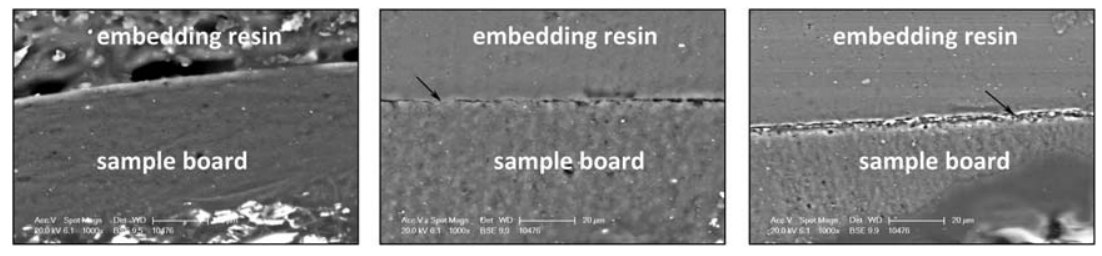

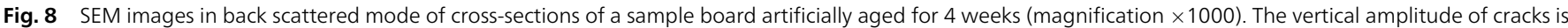

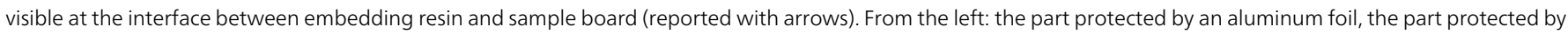
an UV filter, and the uncovered part. 
ageing phenomena. This non-invasive technique can measure micro- and submicrometric roughness (10 to $0.02 \mu \mathrm{m}$ in vertical amplitude). ${ }^{24}$ Thus it is sufficiently sensitive to detect decay producing minute changes in the microtopography of a lacquer's surface at the microscopic level. It provides unambiguous data of the first levels of deterioration of lacquers, before these ones can be identified by a simple visual examination.

Roughness allows us to monitor and quantify the loss of shine of a lacquer during the ageing process, even when it is not visible to the naked eye, as well as the densification of the network of cracks.

\section{Synchrotron radiation micro-FTIR (SR- $\mu$ FTIR)}

4.1 Sample boards. The effect of alteration is clearly shown in the spectra collected from the part of the sample board protected by aluminum and from the uncovered one (Fig. 9). These two kinds of samples are firstly discussed here because they present the higher variations. The light exposure leads to a marked decrease in intensity of the broad band centered at 3400 $\mathrm{cm}^{-1}$ strongly dominated by $\nu \mathrm{OH}$ on the catechol ring. This band can also be assigned to polysaccharides but as they are present in low amounts compared to catechol, their contribution is masked. We also note a decrease in intensity of the shoulder near to $3005 \mathrm{~cm}^{-1}$ and of the band at $1680 \mathrm{~cm}^{-1}$, respectively assigned to $\nu \mathrm{CH}=\mathrm{CH}$ in the side chain and possibly $\nu \mathrm{C}=\mathrm{O}$ of quinone $/ \nu \mathrm{C}=\mathrm{C}$ in the side chain. Concomitantly, the absorption at $1725 \mathrm{~cm}^{-1}$ shifts to $1713 \mathrm{~cm}^{-1}$, and this indicates the formation of oxidation products. The increase of the band near $1045 \mathrm{~cm}^{-1}$ ( $\nu \mathrm{COC}$ and $\mathrm{COH}$ of polysaccharides) suggests that higher fractions of durable polysaccharides are analyzed with the process of degradation., ${ }^{4,25,26}$

As it was difficult to quantify the evolution of bands at 1270 , 1210 and $990 \mathrm{~cm}^{-1}$ by a simple examination of IR spectra, we calculated the area ratios of these bands. Finally, Fig. 10 summarizes the relative areas for the part of the sample board protected by aluminum and for the uncovered one. According to

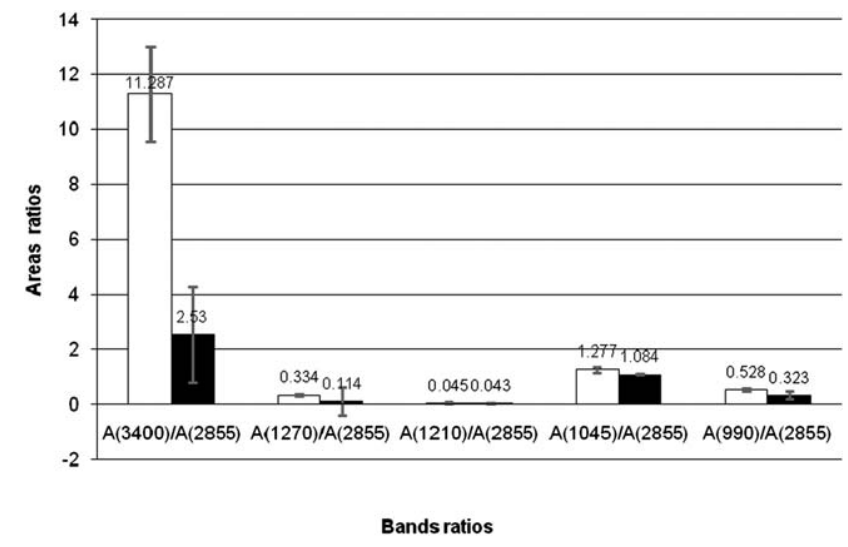

Fig. 10 Area ratios issued from SR micro-FTIR spectra of the part of the sample board protected by an aluminum foil and the uncovered part after an artificial ageing for 4 weeks. Values are the means calculated from five spectra. Standard deviations are reported. In white: the part protected by an aluminum foil and in black: the uncovered part label.

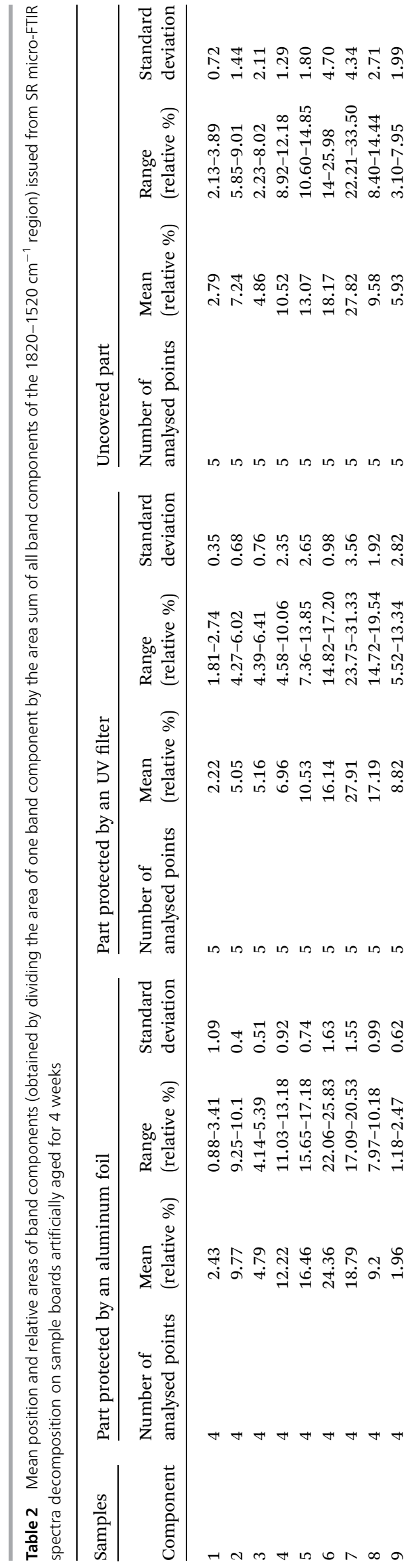


these results, we can note a decrease in the area of the band at $1270 \mathrm{~cm}^{-1} .^{27}$ This band attributed to $\mathrm{C}-\mathrm{OH}$ of catechol exhibits the same behavior as the broad band at $3400 \mathrm{~cm}^{-1}$ which is assigned to $\nu \mathrm{OH}$. Simultaneously, the area of the band at $1210 \mathrm{~cm}^{-1}$, assigned to $=\mathrm{C}-\mathrm{O}-\mathrm{C}$ aromatic ether, remains unchanged. The marked decrease in the band area at $990 \mathrm{~cm}^{-1}$, ascribed to the $\nu \mathrm{CH}$ out of plane of the conjugated triene in the side chain, is interpreted as a decrease in the number of unsaturated side chains. ${ }^{8}$

Table 2 details the mean position and the relative areas of band components issued from the decomposition of the 1820$1520 \mathrm{~cm}^{-1}$ region. As this procedure can highlight detailed and slight variations of the molecular structure, it was applied to the part of the sample board protected by aluminum, the one protected by an UV filter and the uncovered one. An attribution of IR bands is also discussed although it is difficult to ensure a complete and accurate assignment due to the chemical complexity of lacquer and the absence of reference IR spectra of all its individual components (glycoproteins, polysaccharides, catechol, dimeric catechol, etc.). The evolution of the part of the sample board protected by an UV filter and the uncovered one show the same trends during the exposure except for band 8 after the artificial ageing (Fig. 11). It indicates that an alteration of lacquer even occurs by irradiation with visible and infrared light. We can note a decrease in the relative area of bands 5 and 6 issued from the decomposition and in contrast an increase in the relative area of bands 7 and $9(\nu \mathrm{C}=\mathrm{O}$ of ketone, aldehyde or carboxylic acid) comparatively to the part of the sample board protected by aluminum.

As results of the accelerated ageing tests, three alteration phenomena can be predicted. Under light exposure, oxidation products appear on the reactive side chains of catechols. The increase in the relative area of bands 7 and 9 , attributed to $\mathrm{C}=\mathrm{O}$ of ketone, aldehyde and carboxylic acids, correlated with the decrease in intensity of the shoulder near $3005 \mathrm{~cm}^{-1}$ and of the band at $990 \mathrm{~cm}^{-1}$, relative to double bonds from the side chain, are the main evidence of this photooxidation. In fact these $\mathrm{CH}$ unsaturated groups react by integrating oxygen and thus leading to the formation of oxidation products. Moreover the high increase in the relative area of the band at $1740 \mathrm{~cm}^{-1}$ for the part of the sample board protected by an UV filter is surprising. It can suggest that a significant formation of aldehyde has occurred.

Another mechanism can be considered. The photo-oxidation of lacquer also leads to a loss of hydroxyl functions on the catechol ring (decrease in intensity of bands at 3400 and $1270 \mathrm{~cm}^{-1}$ ).

In addition, decomposition of the polymeric network also occurs. The decrease in the relative area of the bands assigned to $\mathrm{C}=\mathrm{C}$ aromatic (bands 2 and 5 issued from the decomposition) indicates a disappearance of the fraction of catechol. Simultaneously the light damage causes an increase in intensity of the band at $1045 \mathrm{~cm}^{-1}$ due to polysaccharides. It evidences that a higher exposure duration corroborates the decrease of the catechol fraction and the increase of the polysaccharide signal. ${ }^{4}$ It means that the densely packed grain structure of the lacquer film, named the core-shell structure (Fig. 2), is damaged during ageing. As the lacquer surface deteriorates, the polymerized catechol is no more protected by polysaccharides and it is decomposed. Concomitantly the durable polysaccharides exhibit a more important IR signal. These chemical changes can be correlated with the microstructural deterioration of the lacquer surface. The polysaccharide grains are visible in the SEM image obtained from the non-protected sample board exposed to light for 4 weeks (Fig. 7, right).

Finally the image of the spatial distribution of the oxidation products is reported in Fig. 12. The oxidation products are clearly located in an area ranging between 15 and $20 \mu \mathrm{m}$ from the surface which corresponds to the thickness of the alteration

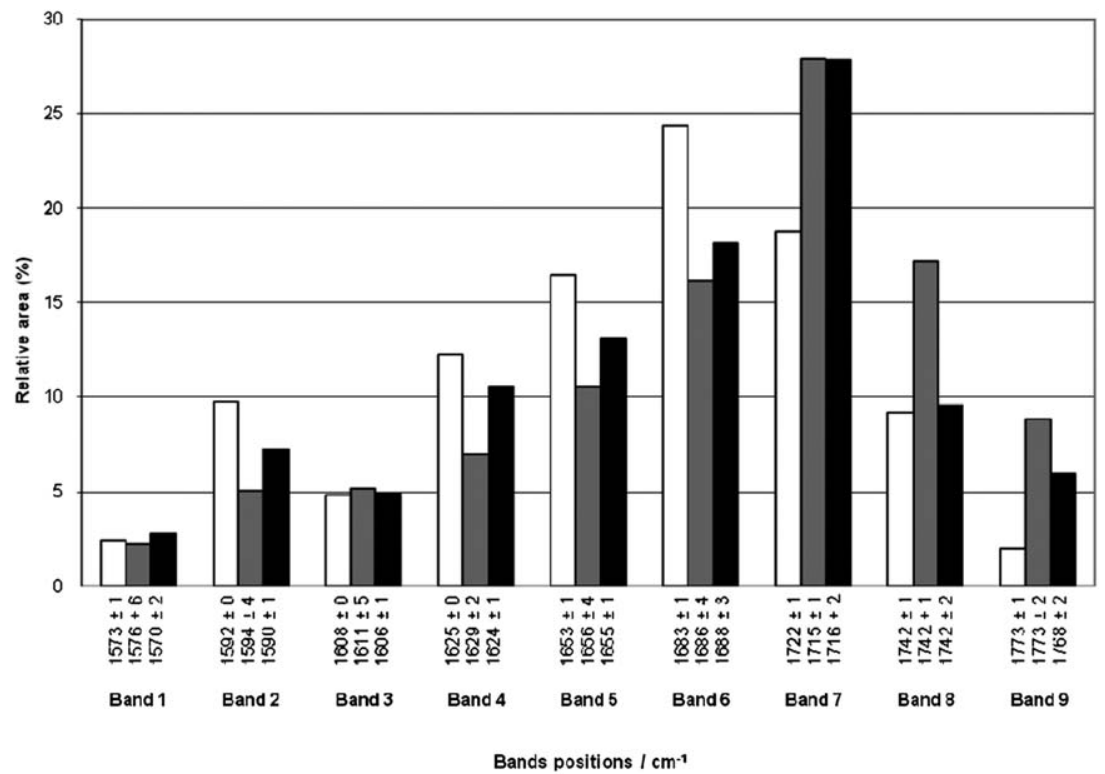

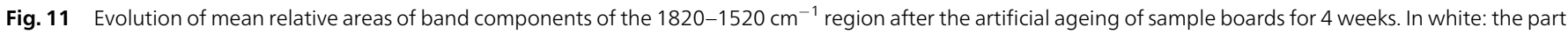
protected by an aluminum foil, in grey: the part protected by an UV filter, and in black: the uncovered part. 

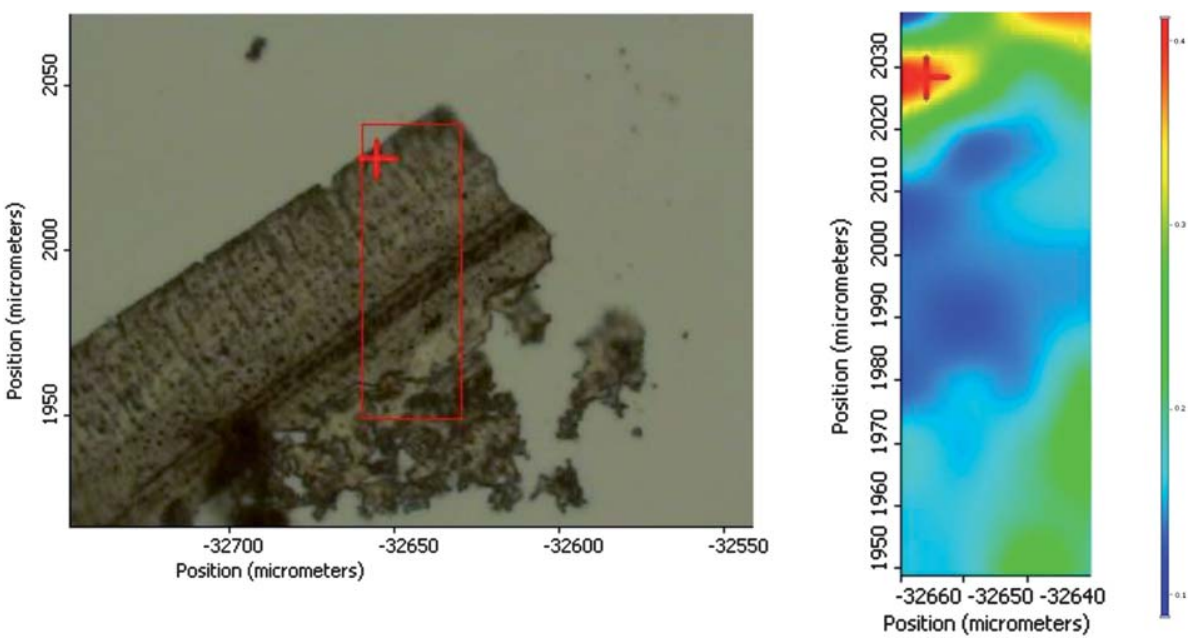

Fig. 12 Optical image of the uncovered part of a sample board artificially aged for 4 weeks and an SR micro-FTIR image of the band at $1716 \mathrm{~cm}^{-1}$ showing the spatial distribution of the oxidation products. A color scale from red (high intensity) to blue (low intensity) was adopted for all images. Images obtained from the microscope Continuum XL (Thermo Nicolet) at the SMIS beamline (synchrotron Soleil) and showing the $10 \times 10 \mu \mathrm{m}^{2}$ analysed area.

front. This map is representative of the degradation state of the lacquer under light exposure.

4.2 Archaeological lacquer. The IR spectra collected from thin sections of archaeological lacquer present the same profile as the one issued from the uncovered part of the sample board, even though band broadenings are also observed (Fig. 9).

However, one can note that the band due to the conjugated trienes in the side chain (at $990 \mathrm{~cm}^{-1}$ ) has completely disappeared, indicating that they have totally reacted to form oxidation products.

These results are in agreement with the conclusions drawn from the aged sample boards. But they underline a higher oxidation state of the archaeological lacquer; as expectedly oxidation of the side chains appears to be complete for a 2000 year period.

\section{Conclusion}

The present work demonstrates that deeper insight into decay mechanisms of Asian lacquer can be obtained by performing a comprehensive physico-chemical characterization. A visualoptical inspection correlated with a SEM examination, microtopography and SR micro-FTIR has permitted the evaluation of the degradation state and the conservation state of Asian lacquer samples. An investigation of modern multilayered lacquers artificially aged by light irradiation and archaeological lacquer was carried out using this combined approach.

Very fine changes of the lacquer microstructure and molecular composition could be detected and are in agreement with macroscopic observations. The light exposure causes a damage of the closely packed shell (polysaccharides-glycoproteins)-core (polymerized catechol) structure of lacquer with the appearance of the polysaccharides which causes a decrease of the surface brightness. The degradation starts from the surface of lacquer. The measurements of roughness by microtopography allowed us to quantify the deterioration using the three-dimensional root-mean square deviation of the surface parameter, $S_{\mathrm{q}}$. From a chemical point of view, the alteration leads to the oxidation of the conjugated side chain of catechol and subsequently to the formation of aldehyde, ketone and carboxylic acid, to a loss of hydroxyl groups on the catechol ring and finally, in the most advanced degradation state, to the decomposition of the polymerized lacquer network.

These changes can also be evidenced by irradiation with visible and IR light, showing that UV radiations are not the only cause of the damages.

The front of degradation of artificially aged lacquer can be imaged at the microscale as well as a more than 2000 years old archaeological lacquer by using SR micro-FTIR.

Thus the proposed approach proved to be a powerful non- or microdestructive methodology to precisely evaluate and depict the state of alteration of Asian lacquer at the macro-, micro- and even molecular scale. It is perfectly adapted to the investigation of the processes involved in the ageing of the cultural heritage.

\section{Acknowledgements}

The authors are grateful to Jean-Jacques Ezrati from C2RMF for guidance about microtography. The artificial ageing was carried out at the Centre de Recherche sur la conservation des collections (CRCC, MNHN, USR 3224 CNRS, Paris, France). The authors thank Bertrand Lavédrine, Martine Gillet and Chantal Garnier for their essential help and advices during these tests. Soleil Synchrotron is acknowledged for having provided beamtime at SMIS beamline through the project number: 20090527. Thanks to Ibraheem Youssef for his assistance. Special thanks go to Martine Rey, an artist lacquer, Anne Jacquin, a lacquer restorer, for supporting sample boards as well as for encouragement and suggestions and also to Danielle Jaillard from CCME (UMR 8080-8620, Orsay) for the preparation of thin sections and her dedication. This research was supported by the PNRCC-MRT program 2008 "Développement d'une méthodologie analytique pour la caractérisation, la compréhension des mécanismes d'altération et la conservation des laques asiatiques issues de contexte archéologique et muséal" from the Ministère de la Culture et de la Communication. 


\section{References}

1 M. Webb, Lacquer: Technology and Conservation, Butterworth Heinemann, Oxford, 2000, p. 182.

2 J. Kumanotani, Prog. Org. Coat., 1995, 26, 163.

3 A.-S. Le Hô, M. Regert, C. Duhamel, O. Marescot, J. Langlois and M. Sablier, Proceedings of Science for Conservation of Cultural Heritage, 2012, French-Japanese Workshop, Paris, Hermann, November 4-5, 2010, pp. 105-118.

4 C. Jin, C. Sano and J. Jumanotani, in East Asian and European Lacquer Techniques, ed. M. Kühlenthal, Bayerischen Landesamt für Denkmalpflege, Munich, 2000, pp. 149-160.

5 S. Rivers, Proceedings of Urushi 2003, International course on conservation of Japanese lacquer, Tokyo, December 3-5, 2003, pp. 150-158.

6 N. Schellmann, in East Asian Lacquer, ed. S. Rivers, R. Faulkner and B. Pretzel, Archetype Publications Ltd. in association with the Victoria and Albert Museum, London, 2011, pp. 107-120.

7 T. Ogawa, K. Arai and S. Osawa, J. Polym. Environ., 1998, 6(1), 59.

8 J.-W. Hong, M.-Y. Park, H.-K. Kim and J.-O. Choi, Bull. Korean Chem. Soc., 2000, 21(1), 61.

9 E. Obataya, Y. Furuta, Y. Ohno, M. Norimoto and B. Tomita, J. Appl. Polym. Sci., 2002, 83, 2288.

10 Y. Kamiya, R. Lu, T. Kumamoto, T. Honda and T. Miyakoshi, Surf. Interface Anal., 2006, 38, 1311.

11 B. Keneghan, in East Asian Lacquer, ed. S. Rivers, R. Faulkner and B. Pretzel, Archetype Publications Ltd. in association with the Victoria and Albert Museum, London, 2011, pp. 51-59.

12 A. E. Elmahdy, P. D. ruiz, R. D. Wildman, J. M. Huntley and S. Rivers, in East Asian Lacquer, ed. S. Rivers, R. Faulkner and
B. Pretzel, Archetype Publications Ltd. in association with the Victoria and Albert Museum, London, 2011, pp. 75-82.

13 X. Liu, A. E. Elmahdy and R. D. Wildman, in East Asian Lacquer, ed. S. Rivers, R. Faulkner and B. Pretzel, Archetype Publications Ltd. in association with the Victoria and Albert Museum, London, 2011, pp. 83-91.

14 C. Mc Sharry, R. Faulkner, S. Rivers, M. S. P. Shaffer and T. Welton, Rev. Conserv., 2007, 8, 29.

15 A.-S. Le Hô, M. Regert, O. Marescot, C. Duhamel, J. Langlois, T. Miyakoshi, C. Genty and M. Sablier, Anal. Chim. Acta, 2012, 710, 9.

16 J. Filik, M. D. Frogley, J. K. Pijanka, K. Wehbe and G. Cinque, Analyst, 2012, 137, 853.

17 P. Bassan, J. Lee, A. Sachdeva, J. Pissardini, K. M. Dorling, J. S. Fletcher, A. Henderson and P. Gardner, Analyst, 2013, 138, 144.

18 C. Paris, S. Lecomte and C. Coupry, Spectrochim. Acta, Part A, $2005,62,532$.

19 M. Lebon, I. Reiche, F. Fröhlich, J. J. Bahain and C. Falguères, Anal. Bioanal. Chem., 2008, 392, 1479.

20 C. Chadefeaux, A.-S. Le Hô, L. Bellot-Gurlet and I. Reiche, $e$ Preserv. Sci., 2009, 6, 1.

21 C. Daher, C. Paris, A.-S. Le Hô, L. Bellot-Gurlet and J.-P. Echard, J. Raman Spectrosc., 2010, 41, 1204.

22 C. Daher, L. Bellot-Gurlet, A.-S. Le Hô, C. Paris and M. Regert, Talanta, 2013, 115, 540.

23 J. Kumanotani, Prog. Org. Coat., 1998, 34, 135.

24 C. Minet, PhD thesis, Poitiers University, 2009, p. 194.

25 J. Yang, Y. Du, R. Huang, Y. Wan and T. Li, Int. J. Biol. Macromol., 2002, 31, 55.

26 J. Yang and Y. Du, Carbohydr. Polym., 2003, 52, 405.

27 J. Xia, Y. Xu, J. Lin and B. Hu, Prog. Org. Coat., 2008, 61, 7. 